\section{EXATAS E TECNOLÓGICAS}

V.4 • N.1 2020 - Fluxo Contínuo

ISSN Digital: 2359-4942

ISSN Impresso: 2359-4934

DOI: $10.17564 / 2359-4942.2020 v 4 n 1$

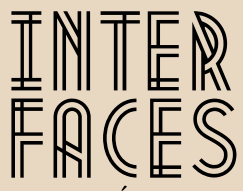

CIENTÍFICAS

\title{
IDENTIFICAÇ̃̃O DE FIBRILAÇÃO ATRIAL BASEADA NOS INTERVALOS RR E COMPLEXO QRS DO SINAL ECG
}

IDENTIFICATION OF ATRIAL FIBRILLATION BASED IN ECG RR INTERVALS AND QRS COMPLEX

IDENTIFICACIÓN DE LA FIBRILACIÓN AURICULAR BASADA EN EL INTERVALO RR Y COMPLEJO QRS DE LAS SEÑALES DE EKG

Victor Gabriell Ribeiro da Silva Dami Dória Narayana Duarte ${ }^{2}$

\section{RESUMO}

Uma porção considerável das mortes em todo planeta são provocadas por doenças no coração, como infarto agudo do miocárdio e arritmias cardíacas, diagnosticar estas cardiopatias é de extrema importância para a saúde do paciente. Visando acelerar a identificação de cardiopatias, diversos procedimentos têm sido desenvolvidos com intuito de reconhecer automaticamente estas doenças por meio do sinal eletrocardiográfico (ECG). Este trabalho apresenta uma metodologia para reconhecer fibrilação atrial (FA) por meio de sinais ECG, inicialmente o sinal é pré-processado, em seguida são extraídas características baseadas na entropia aproximada e análise estatística dos intervalos RR e do complexo QRS, ao final, uma rede neural artificial é utilizada para classificar os sinais em normais ou com FA. O trabalho concluiu que o método é capaz de diferenciar os dois tipos de sinais com acurácia total de 98,1\%.

\section{PALAVRAS-CHAVE}

Eletrocardiograma. Extração de Características. Fibrilação Atrial. Rede Neural Artificial. 


\section{ABSTRACT}

A considerable portion of deaths worldwide are caused by heart disease, such as acute myocardial infarction and cardiac arrhythmias, diagnosing these diseases is very important for the patient's health. In order to speed up the identification of heart diseases, several procedures have been developed in order to automatically recognize these diseases through the electrocardiographic (ECG) signal. This paper presents a methodology to recognize atrial fibrillation (AF) through ECG signals, initially the signal is pre-processed, then features based on approximate entropy and statistical analysis of RR intervals and QRS complex are extracted, at the end, an artificial neural network is used to classify the signals as normal or with AF. The study concluded that the method is capable of recognizing between the two types of signals with accuracy of $98.1 \%$.

\section{KEYWORDS}

Atrial fibrillation. Artificial neural network. Electrocardiogram. Feature Extraction.

\section{RESUMEN}

Una porción considerable de las muertes en todo el planeta es provocada por enfermedades en el corazón, como infarto agudo de miocardio y arritmias cardíacas, diagnosticar estas cardiopatías es de extrema importancia para la salud del paciente. Para acelerar la identificación de enfermedades del corazón, se han desarrollados varios procedimientos para reconocer automáticamente estas enfermedades a través de la señal electrocardiográfica (EKG). Este trabajo presenta una metodología para reconocer la fibrilación auricular (FA) mediante señales de EKG, inicialmente la señal se procesa previamente, luego se extraen las características basadas en la entropía aproximada y el análisis estadístico de los intervalos RR y el complejo QRS, al final una red neural artificial se utiliza para clasificar las señales como normales o con FA. El trabajo concluyó que el método es capaz de diferenciar los dos tipos de señales con una precisión total de $98.1 \%$.

\section{PALABRAS-CLAVE}

Electrocardiograma. Extracción de características. Fibrilación auricular. Red neuronal artificial.

Interfaces Científicas • Aracaju • V.4 • N.1 • p. 9 - 20 • 2020 • Fluxo Contínuo 


\section{INTRODUÇ̧̃̃o}

Uma das principais metodologias para o diagnóstico clínico de doenças cardíacas é por meio da análise do sinal eletrocardiográfico (ECG), sendo este uma das formas mais simples, barata e não invasiva de se identificar cardiopatias. O sinal ECG é uma representação da atividade elétrica do coração, provocada pela despolarização e repolarização dos átrios e ventrículos (ROOPA; HARISH, 2017).

De acordo com Yildirim e outros autores (2018), a eletrocardiografia é a técnica mais básica e acessível para obter o registro da atividade elétrica cardíaca, esta pode indicar arritmias cardíacas, como a fibrilação atrial (FA). É de extrema importância que estes problemas sejam detectados o mais rápido possível a fim de evitar graves complicações para o paciente, além disto as doenças cardíacas são as que mais matam em todos planeta (RITCHIE; ROSER, 2018).

Segundo Kirchhof e outros autores (2016), é uma forma de arritmia cardíaca, sendo uma das principais causas de acidente vascular cerebral e insuficiência cardíaca, estudos afirmam que aproximadamente $3 \%$ da população adulta de todo planeta possui FA, especialmente em pessoas mais velhas, com hipertensão, obesidade ou com diabetes mellitus.

Diversas metodologias têm sido investigadas para a detecção automática da FA por meio do sinal ECG; algumas destas são baseados na análise morfológica do sinal ECG (MONDÉJAR-GUERRA et al., 2019) e na análise dos intervalos RR (BILLECI et al., 2017). Diversos trabalhos têm utilizado redes neurais artificiais ou máquina de vetor de suporte como classificadores, obtendo resultados promissores (BERKAYA et al., 2018).

O presente trabalho visa classificar automaticamente a fibrilação atrial por meio de sinais ECG de curta duração (10 s), para isto, o sinal é processado, em seguida são extraídas características do sinal ECG e estas são enviadas para uma rede neural artificial, que irá decidir de acordo com as informações recebidas se o sinal é normal ou com fibrilação atrial.

Os sinais cardíacos usados neste trabalho são do banco de dados do MIT-BIH Normal Sinus Rhythm Database (NSRDB) e o MIT-BIH Arrhythmia database (MITDB), ambos elaborados por Moody e Mark (2001). Estes sinais fazem partes do banco de dados da plataforma PhysioNet desenvolvido por Goldberger e outros autores (2000).

\section{METODOLOGIA}

A metodologia utilizada no presente trabalho é dividida em três etapas fundamentais: pré-processamento, extração de características e classificação. Na Figura 1 é apresentado o fluxograma da metodologia proposta. 
Figura 1 - Fluxograma da metodologia utilizado neste trabalho

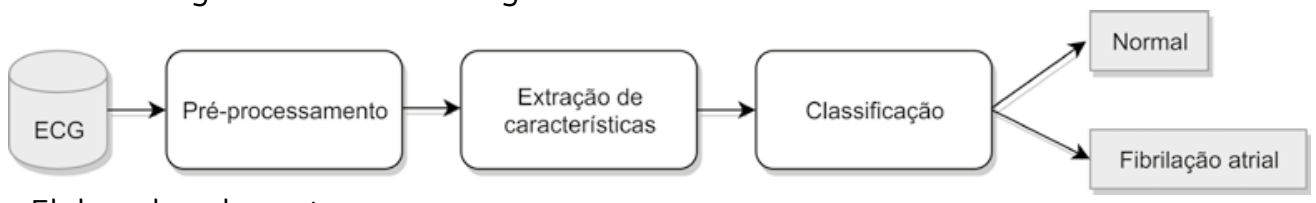

Fonte: Elaborado pelos autores.

\subsection{PRÉ-PROCESSAMENTO}

A etapa de pré-processamento dos dados é realizada na maioria dos sistemas relacionados à extração de características do sinal eletrocardiográfico. 0 principal objetivo desta etapa é melhorar a qualidade do sinal, a fim de tornar determinadas características mais visíveis (JAMBUKIA; DABHI; PRAJAPATI, 2015).

No presente trabalho, o pré-processamento é responsável pela filtragem e remoção do nível DC do sinal ECG. Isto é necessário, pois o sinal obtido do banco de dados do NSRDB e MITDB possui alto nível de sinal DC e de ruído, a redução destes facilita a etapa de extração de características. Para a remoção do nível DC é utilizado a Equação 1, em que o sinal x[n] (sinal ECG) com N amostras é subtraído de sua média para obter saída y[n] (sinal sem nível DC).

$$
y[n]=x[n]-\frac{1}{N} \sum_{i=0}^{N} x[i] \quad(1)
$$

No item a da Figura 2 é mostrado um sinal ECG e no item $\mathbf{b}$ o mesmo sinal agora sem o nível DC. A média do sinal do item a é 970,02, já a média do sinal do item b é 1,6674e-14, aproximadamente zero. Este procedimento é bastante útil para extração de características, discutida a seguir.

Figura 2 - Item (a) Sinal ECG com nível DC e item (b) o mesmo sinal sem nível DC

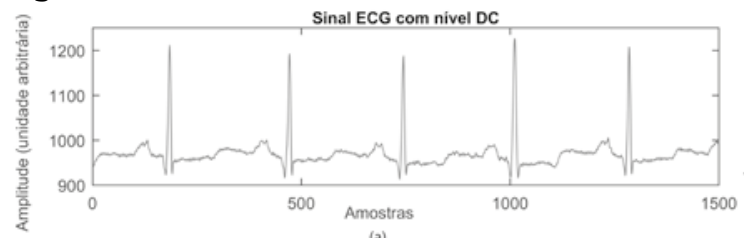

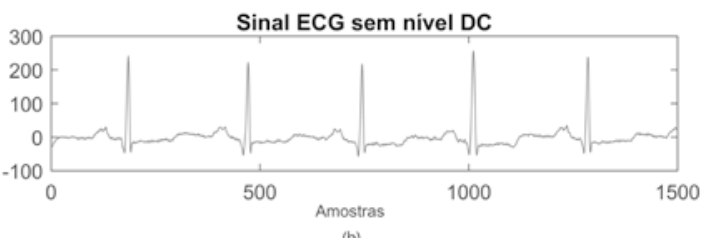

Fonte: Elaborado pelos autores.

Após a remoção do nível DC o sinal ECG é filtrado com um filtro digital FIR passa-banda com frequência de corte entre 1,5 e $45 \mathrm{~Hz}$, este é necessário para reduzir o nível de ruído de baixa e alta frequência presente no sinal.

\subsection{EXTRACÃ̃ DE CARACTERÍSTICAS}

A etapa de extração de características é uma das etapas essenciais na área de reconhecimento de padrões, uma vez que é a responsável por extrair informação dos dados brutos e permitir que o classificador seja capaz de identificar diferenças entre determinados padrões (GARCIA, 2016). 
No presente trabalho, três informações são extraídas dos sinais ECG: entropia aproximada - EnAp, do vetor de intervalos RR (intervalo entre uma onda R e outra), desvio padrão do vetor de intervalos RR e média do vetor de curtose do complexo QRS. Cada uma destas características é discutida a seguir.

Inicialmente é necessário identificar as ondas R no sinal ECG, para isto, é utilizado um algoritmo que analisa todo o sinal e reconhece seus maiores valores, de forma que picos que sejam superiores a um determinado limite e possuam uma determinada proeminência são considerados ondas $\mathrm{R}$. Sendo assim é possível obter um vetor com a posição de todas as ondas R no sinal ECG. Para obter o vetor de intervalos RR basta somente subtrair cada amostra do vetor com a posição das ondas R pela amostra anterior. Em posse do vetor de diferença, é possível calcular a EnAp e o desvio padrão deste vetor.

$\mathrm{Na}$ teoria da informação, a entropia determina o nível de incerteza que a saída de determinado sistema pode ter, desta forma, considerando um conjunto de eventos e que a probabilidade de cada evento acontecer é conhecida, podemos então definir o quanto desconhecido será a escolha de cada evento (BEN-NAIM, 2019).

Um novo conceito de entropia foi apresentado no trabalho de Pincus (1995): entropia aproximada, esta é muito utilizada para medir o grau de irregularidade (ou incerteza) de um determinado sistema. A utilização da EnAp em sinais ECG pode ser muito proveitosa no processo de extração de características. $O$ algoritmo utilizado neste trabalho para determinar a EnAp foi baseado no trabalho elaborado por Pincus (1995), utilizando $m=2$ e $r=3$.

Determinar a EnAp do vetor de intervalos RR do sinal ECG é bastante importante, uma vez que em sinais de fibrilação atrial normalmente existe uma irregularidade nos intervalos entre cada onda $R$, ao contrário de sinais normais, em que os intervalos são aproximadamente constantes, como é mostrado no trabalho de Nascimento e Mesquita (2010). Na Figura 3 é apresentado um sinal considerado normal e outro com fibrilação atrial, em que é possível visualizar a diferença entre o ritmo cardíaco dos dois sinais.

Figura 3 - (a) Sinal ECG normal e (b) Sinal ECG com fibrilação atrial

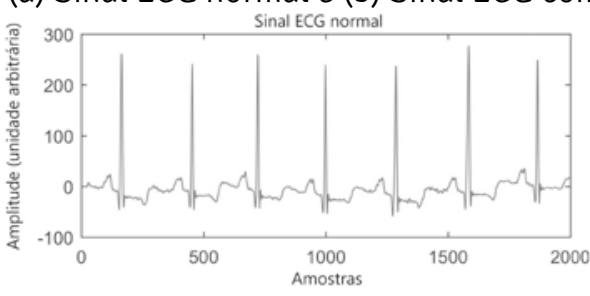

(a)

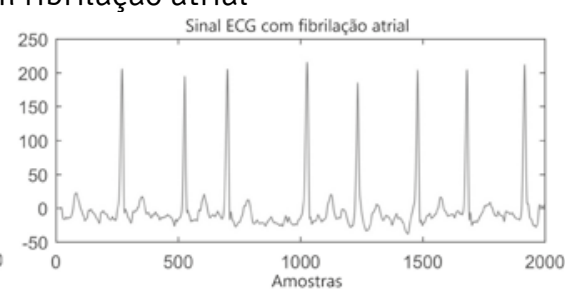

(b)

Fonte: Elaborado pelos autores.

Além da EnAp, também é calculado o desvio padrão (DP) do vetor de intervalos RR. O DP é uma medida de dispersão de dados, indicando o quanto determinadas amostras estão distantes da média. Esta medida é importante, pois é capaz de medir o nível de irregularidade dos intervalos entre cada onda R. $O$ desvio padrão ${ }^{f_{n}}$ : alculado como mostrado na Equação 2, em que $x$ é o sinal de entrada (vetor de intervalos RR), $\bar{x} \bar{x}$ é a média deste sinal e $\mathrm{N}$ é o número total de amostras do sinal $\mathbf{x}$. 


$$
s-\sqrt{\sum_{i=1}^{N}(x[i]-\bar{x})^{2}}
$$

Além de extrair informações associadas ao vetor de intervalos entre as ondas $\mathrm{R}$, também é extraído uma característica relacionada ao complexo QRS do sinal ECG. Este complexo é definido como o momento entre a onda $\mathrm{Q}$ e a onda S, como mostrado na Figura 4.

Figura 4 - (a) Complexo QRS de um sinal ECG normal e (b) Complexo QRS de um sinal com fibrilação atrial
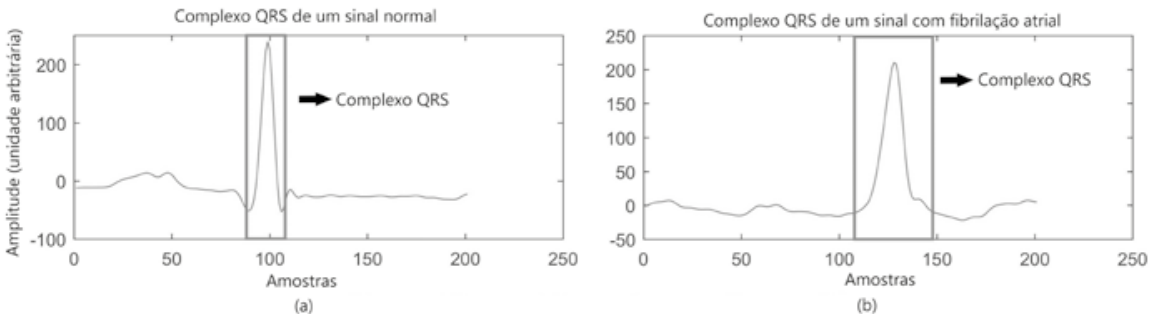

Fonte: Elaborado pelos autores.

Analisar o complexo QRS é bastante importante, visto que este apresenta informações relevantes a respeito do sinal cardíaco, além disto, o complexo QRS de um sinal com fibrilação atrial é consideravelmente diferente do complexo de um sinal normal, na Figura 4 é possível observar esta diferença. Para identificar a região do complexo QRS é utilizado o vetor de posições das ondas R do sinal ECG, sabendo que a onda $\mathrm{R}$ é a região central do complexo QRS foi utilizado para análise todo o período compreendido entre 83 ms antes e depois da onda R, como é ilustrado na Figura 5.

Figura 5 - Determinação da região próxima ao complexo QRS a ser analisada

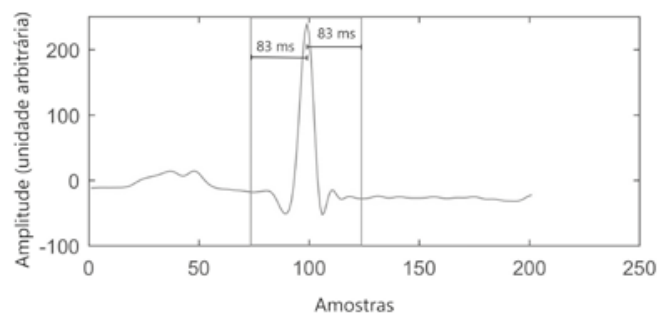

Fonte: Elaborado pelos autores.

Realizada a identificação da região próxima ao complexo QRS, é então calculada a curtose desta parte do sinal. A curtose é uma medida de dispersão que indica o achatamento de uma determinada distribuição. É calculado a curtose de cada região próxima do complexo QRS do sinal ECG, obtendo um vetor com os valores de curtose para cada região, em seguida é calculada a média desse vetor.

Outros valores foram testados para o comprimento da região próxima ao complexo QRS, entretanto com a janela de $83 \mathrm{~ms}$ foram obtidos os melhores resultados na separação entre sinais normais e com fibrilação atrial, calculando a curtose desta região, como é mostrado na Figura 7. 
No final do processo de extração de características, é obtido um vetor com três informações para cada sinal ECG. A seguir, a Figura 6 ilustra o procedimento para extração de características proposto no presente trabalho. As siglas no vetor de características estão relacionadas aos extratores utilizados, significando: EnAp - Entropia aproximada dos intervalos RR, DPRR - Desvio padrão dos intervalos RR e MCQRS - Média do vetor de curtose do QRS.

Figura 6 - Fluxograma da metodologia de extração de características

Fonte: Elaborado pelos autores.

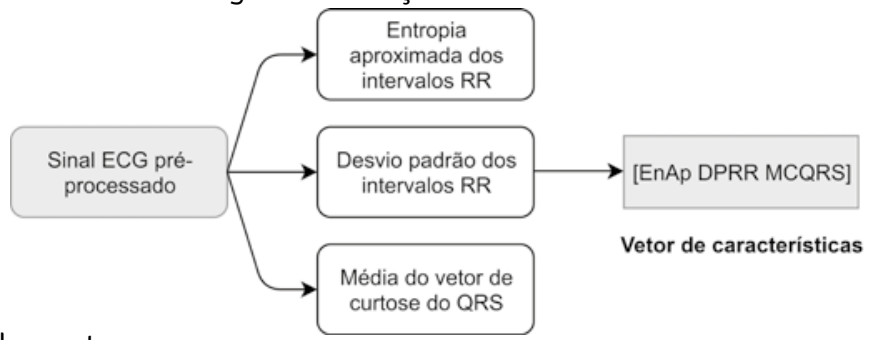

Na Figura 7, é apresentada a distribuição no espaço das três características extraídas (EnAp, DPRR e MCQRS), descritas anteriormente. Na Figura é possível observar que as três características extraídas permitem separar os sinais normais dos sinais com fibrilação atrial.

Figura 7 - Distribuição das características extraídas dos sinais ECG normais (vermelho) e com fibrilação atrial (azul)
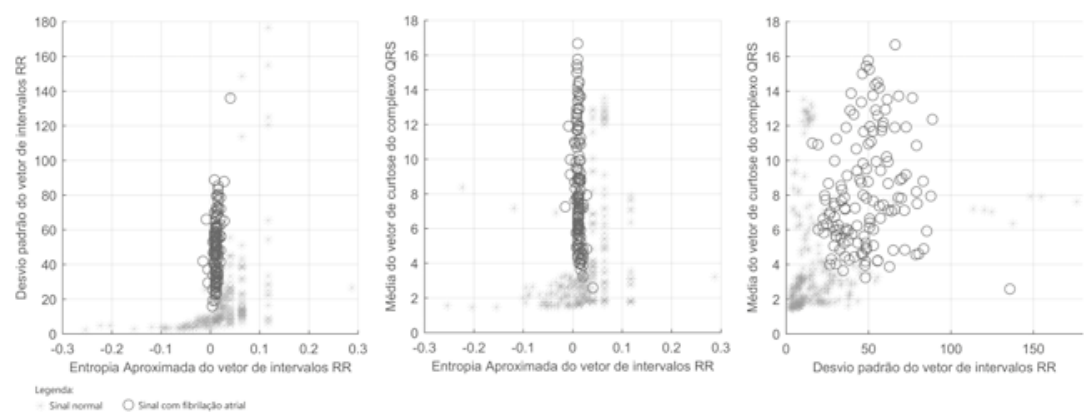

Fonte: Elaborado pelos autores.

\subsection{CLASSIFICAÇÃO}

Para a classificação dos sinais ECG em normais ou com fibrilação atrial, foi utilizada uma rede neural artificial (RNA), esta é um método de aprendizado de máquina inspirado no funcionamento do cérebro humano. De acordo com Suterio (2017), a RNA pode ser definida por um conjunto de unidades de processamento, caracterizado por neurônios artificiais, interligados pelas sinapses artificiais que são representadas por vetores ou matrizes de pesos sinápticos. 
A RNA utilizada neste trabalho possui uma camada de entrada (com três entradas, referente ao número de características extraídas), uma camada oculta (com 10 neurônios, número escolhido arbitrariamente) e uma camada de saída (com duas saídas, referente ao número de classes), como ilustrado na Figura 8.

No total 421 sinais eletrocardiográficos foram analisados, sendo 285 sinais considerados normais e 136 com fibrilação atrial. Os dados foram separados entre treinamento (70\%), validação (15\%) e teste (15\%), desta forma o teste é feito com dados diferentes dos que foram treinados, isto é importante para evitar o sobreajuste (conhecido como overfitting).

Figura 8 - Arquitetura da RNA utilizada neste trabalho

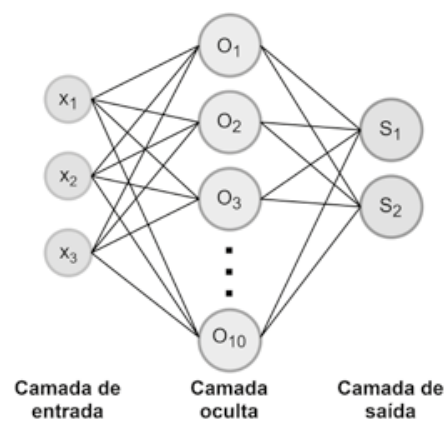

Fonte: Elaborado pelos autores.

\section{RESULTADOS E DISCUSSÕES}

Os resultados obtidos com a utilização da metodologia proposta neste trabalho são apresentados na matriz de confusão ilustrada na Figura 9. A matriz de confusão relaciona as classes preditas (saída da rede neural) e as classes reais (classes verdadeiras). Os valores na diagonal indicam os acertos (em verde) e os valores fora da diagonal apontam os erros no processo de classificação (em vermelho).

A precisão mostrada na Figura 9 indica a proporção de acertos para cada classe predita e é calculada como mostrado a seguir, em que $V P V P=$ verdadeiro positivo (sinal predito corretamente) e $F P$ $F P=$ falso positivo (sinal predito incorretamente), os subscritos $n n$ e $f f$ indicam normal e fibrilação atrial, respectivamente.

$$
\begin{aligned}
& \text { Procisão (Normal) }-\frac{V P_{n}}{V P_{n}+F P_{n}}-\frac{279}{279+2}-0,993 \\
& \text { Procisão (Fib. A.) }-\frac{V P_{f}}{V P_{f}+F P_{f}}-\frac{134}{134+6}-0,957
\end{aligned}
$$

A sensibilidade indica a proporção de acertos para cada classe real e é calculada como mostrado a seguir, em que $F N F N=$ falso negativo. 


$$
\begin{aligned}
& \text { Sonsibilidade (Normal) }-\frac{V P_{n}}{V P_{r}+F N_{n}}-\frac{279}{279+6}-0,979 \\
& \text { Sensibilidade (Fib. A.) }-\frac{V P_{f}}{V P_{f}+F N_{f}}-\frac{134}{134+2}-0,985
\end{aligned}
$$

A proporção no bloco azul (canto inferior direito) indica a acurácia total da rede neural e é calculada como mostrado na Equação 5, em que $V N=$ verdadeiro negativo.

$$
\text { Acurácia total }=\frac{V P_{n}+V P_{a}}{V P_{n}+V P_{f}+F N_{n}+F N_{f}}=\frac{279+134}{279+134+6+2}=0,981
$$

\begin{tabular}{|c|c|c|c|c|}
\hline \multicolumn{5}{|c|}{ Classe real } \\
\hline \multirow{4}{*}{ 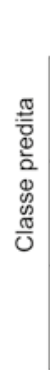 } & & Normal & Fib. A. & Precisão \\
\hline & $\begin{array}{l}\overline{\widetilde{\widetilde{\sigma}}} \\
\text { है } \\
\text { z }\end{array}$ & 279 & 2 & $99,3 \%$ \\
\hline & $\begin{array}{l}< \\
\text { 这 }\end{array}$ & 6 & 134 & $95,7 \%$ \\
\hline & $\begin{array}{l}\frac{0}{\overline{0}} \\
\frac{\omega}{\Phi} \\
\infty\end{array}$ & $97,9 \%$ & $98,5 \%$ & $98,1 \%$ \\
\hline
\end{tabular}

Figura 9 - Matriz de confusão dos resultados obtidos

Fonte: Elaborado pelos autores.

A partir da matriz de confusão apresentada na Figura 8, é possível observar que a rede neural artificial classificou corretamente a maior parte dos sinais ECG, obtendo acurácia total de 98,1\%. A identificação de sinais considerados normais foi a que obteve melhor precisão.

A maior parte dos erros de classificação são provocados por falhas no algoritmo de extração de características, principalmente pela não identificação correta das ondas R nos sinais ECG. Os erros ainda podem acontecer devido aos valores atípicos (outliers) que determinados sinais podem obter durante o processo de extração de características, isto pode ser observado na Figura 6, em que alguns sinais apresentam valores muito diferentes dos outros sinais de mesma classe, tornando-os mais dispersos no espaço de características.

\section{CONCLUSÕES}

O presente trabalho apresenta uma proposta de reconhecimento automático de fibrilação atrial por meio de sinais eletrocardiográficos de curta duração. Três características foram extraídas dos sinais eletrocardiográficos e uma rede neural artificial foi utilizada para classificar os sinais em duas classes: normal e com fibrilação atrial. 
A metodologia exposta obteve bons resultados, atingindo acurácia total de 98,1\%, este bom resultado deve-se a diferenças fundamentais entre sinais normais e sinais com fibrilação atrial, como o ritmo cardíaco e a morfologia do complexo QRS. Além do bom resultado, o método proposto para extração de características é de baixa complexidade computacional, o que facilita sua aplicação em outras plataformas. Determinadas falhas no processo de extração de características (em especial erros associados à não detecção das ondas $\mathrm{R}$ nos sinais eletrocardiográficos) provocaram alguns erros na etapa de classificação.

É importante que em trabalhos futuros sejam capazes de classificar outros tipos de arritmias cardíacas, como o flutter atrial ou contração ventricular prematura. É interessante, também, que as técnicas de extração de características sejam aperfeiçoadas, utilizando técnicas como análise de discriminantes lineares para melhorar a separação entre as classes, podendo tornar ainda mais eficiente a identificação das principais arritmias cardíacas.

É importante, sabendo da relevância do reconhecimento de arritmias cardíacas por meio de sinais ECG, que estudos futuros apresentem, também, técnicas capazes de realizar este processo em tempo real, para que possa ser aplicado em ambientes hospitalares, principalmente em situações de urgência e emergência.

\section{REFERÊNCIAS}

BEN-NAIM, A. Entropy and Information Theory: Uses and Misuses. Entropy, v. 21, n. 12, p. 1170, 2019.

BERKAYA, S. K. et al. A survey on ECG analysis. Biomedical Signal Processing and Control, v. 43, p. 216-235, 2018.

BILLECI, L. et al. Detection of AF and other rhythms using RR variability and ECG spectral measures. Computing in Cardiology (CinC), p. 1-4, IEEE, set. 2017.

\section{GARCIA, G. C. Seleção de características e otimização de parâmetros via PSO para um classificador de arritmias cardíacas. 2016.}

GOLDBERGER, A. L. et al. PhysioBank, PhysioToolkit, and PhysioNet: components of a new research resource for complex physiologic signals. Circulation, v. 101, n. 23, p. e215-e220, 2000.

JAMBUKIA, S. H.; DABHI, V. K.; PRAJAPATI, H. B. Classification of ECG signals using machine learning techniques: A survey. International Conference on Advances in Computer Engineering and Applications, Anais..., IEEE, mar. 2015. p. 714-721.

KIRCHHOF, P. et al. ESC Guidelines for the management of atrial fibrillation developed in collaboration with EACTS. European journal of cardio-thoracic surgery, v. 50, n. 5, p. e1-e88, 2016. 
MONDÉJAR-GUERRA, V. et al. Heartbeat classification fusing temporal and morphological information of ECGs via ensemble of classifiers. Biomedical Signal Processing and Control, v. 47, p. 41-48, 2019.

MOODY, G. B.; MARK, R. G. The impact of the MIT-BIH arrhythmia database. IEEE Engineering in Medicine and Biology Magazine, v. 20, n. 3, p. 45-50, 2001.

NASCIMENTO, B. C. D.; MESQUITA, E. T. Fibrilação atrial na insuficiência cardíaca: novos avanços na genética molecular e no tratamento. Rev. Bras. Cardiol., v. 23, n. 1, p. 25-34, 2010.

PINCUS, S. Approximate entropy (ApEn) as a complexity measure. Chaos: An Interdisciplinary Journal of Nonlinear Science, v. 5, n. 1, p. 110-117, 1995.

RITCHIE, H.; ROSER, M. Our World in Data. Causes of death, published February. 2018.

ROOPA, C. K.; HARISH, B. S. A survey on various machine learning approaches for ECG analysis. International Journal of Computer Applications, v. 163, n. 9, p. 25-33, 2017.

SUTERIO, V. Detecção de cardiopatias por eletrocardiograma utilizando redes neurais artificiais. Bachelor's thesis, Universidade Tecnológica Federal do Paraná. 2017.

YILDIRIM, Ö. et al. Arrhythmia detection using deep convolutional neural network with long duration ECG signals. Computers in biology and medicine, v. 102, p. 411-420, 2018. 
1 Graduado em Engenharia Mecatrônica pela UNIT; Membro do Grupo de pesquisa em Bioengenharia e Medicina.

E-mail: victoribeiro@outlook.com.br

2 Mestre em Engenharia Elétrica pela UFS; membro do Grupo de pesquisa em Bioengenharia e Medicina.

E-mail: damidoriad@gmail.com

\section{(). (1) (2)}

Este artigo é licenciado na modalidade acesso abertosob a Atribuição-Compartilhalgual CC BY-SA

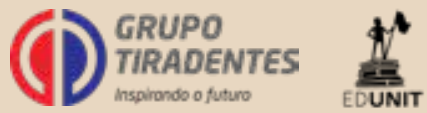

